\title{
Extracting and Segmenting Container Name from Container Images
}

\author{
M. M. Aftab Chowdhury \\ Dept. of CSE,CUET
}

\author{
Kaushik Deb,Ph.D \\ Dept.of CSE,CUET
}

\begin{abstract}
Container name extraction is very important to the modern container management system.Similar techniques have been suggested for vehicle license plate recognition in past decades.Container name extraction has more complexity from license plate extraction because of the severity of nonuniform illumination and invalidation of color information. The main purpose of this paper is to propose a new methodology for text extraction,segmenting text characters and removing non text,background from images. Existing text extraction methods do not work efficiently in case of images with noice and complex background. Documents with only text work efficiently in OCR.The approach used is based on edge detection,close operation,detecting connected components,removing non text regions and character segmentation.
\end{abstract}

\section{General Terms}

Pattern Recognition,Image Processing,OCR,Character Segmentation.

\section{Keywords}

Mathematical Morphology,Morphological Operators,Edge detection,Localization, Connected Component.

\section{INTRODUCTION}

Text extraction is very important for document image analysis,processing[1] and recognition pipeline. Detecting, segmenting, and extracting text from document images which includes text are the most important facts. Image Binarization which converts a gray image (up to 256 gray levels) to black and white image $(0$ or 1$)$ is a basic preprocessing steps[2][3].This process reduces the computational load and the complexity of the algorithm.Mathematical morphology is a powerful tools for extracting geometrical structures and shapes.

Although it seems vehicle license plate extraction and container text extraction is very similar but there are more challenges.In locating license plate region color and plate edges are very useful but these items are not useful for locating license region.Moreover.container name extraction faces difficulties because of outdoor illumination conditions when compared with license plate text extraction.Surface curvature causes severe illumination change for container name characters.For this kind of reasons,methods for license plate extraction are not suitable for container name extraction.
In this paper, we propose an algorithm for text string extraction based on morphological operations. The proposed algorithm is insensitive to skew and text orientation, is free from artifacts that are introduced by both global and fixedsize block-based local thresholding, and robust to noise.

The paper is organized as follows. In Section II, the proposed morphological text extraction and segmentation technique is described. Examples and experimental results are presented in Section III.Comparison with existing techniques in section IV. A conclusion is given in Section V.

\section{METHODOLOGY}

The method has considered the fact that edges are reliable features of text regardless of color or intensity, layout, orientation etc. The edge detection operation is performed using the basic operators of mathematical morphology.Using the edges the algorithm has tried to find out text candidate connected components. These components have been labeled to identify different components of the image. Once the components have been identified, the variance is found for each connected component considering the gray levels of those components. Then the text is extracted by selecting those connected components whose variance is less than some threshold value. The complete process of text extraction and segmentation is given in the form of flow chart in Figure 1.

\subsection{Converting to Gray}

The RGB components of the input color image are combined to give an intensity image $\mathrm{Y}$ as follows:

$\mathrm{Y}=0: 299 \mathrm{R}+0: 587 \mathrm{G}+0: 114 \mathrm{~B}$

where $R ; G$ and $B$ are the red, green and blue components of the input color image, respectively. The color components may differ in a text region (and/or its surrounding background) while having an almost constant intensity. So,the intensity image $\mathrm{Y}$ is processed in the next steps of the algorithm rather than the color components $\mathrm{R} ; \mathrm{G}$ and $\mathrm{B}$.

\subsection{Edge Detection}

For the given input image an efficient morphological edge detection scheme is applied to find the edges of the image. The complete process of edge detection is given in the form of flow chart in Figure 2.

Step 1: The image is blurred (to reduce false edges and oversegmentation) using open-close and close-open filters 
Input Image

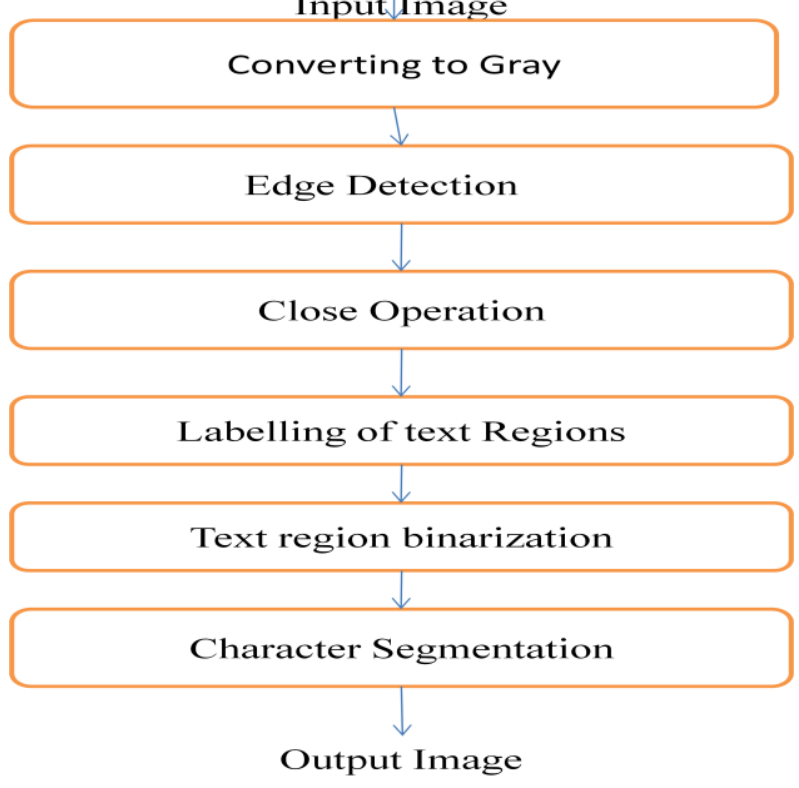

Fig 1: Flow Chart for Text Extraction

The final blurred image is the average of the outputs of these filters.

Step 2: Morphological gradient (MG) operator is applied to the blurred image $\mathrm{Ybl}$ resulting in an image es as follows:

$$
\text { es }=\delta \mathrm{B}(\mathrm{Ybl})-\varepsilon \mathrm{B}(\mathrm{Ybl})
$$

Where, $\mathrm{B}$ is connected structuring element, $\delta \mathrm{B}$ is dilation with $\mathrm{B}$ on $\mathrm{Ybl}, \varepsilon \mathrm{B}$ is erosion with $\mathrm{B}$ on $\mathrm{Ybl}$.

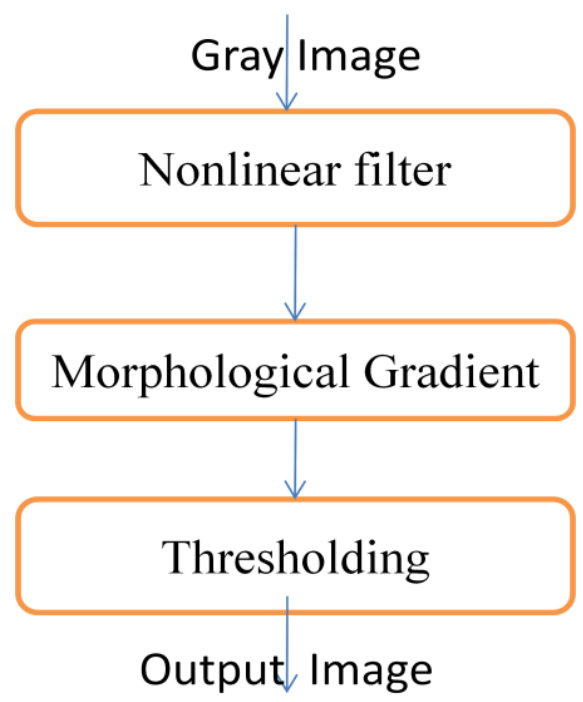

Fig 2:Flow Chart for Edge Detection

Step 3: The resulting image es is then thresholded to obtain a binary edge image. The threshold level $\mathrm{\gamma}$ is determined by $\mathrm{\gamma}=\frac{\sum(e s . s)}{\sum s}$

where "." denotes pixel-wise multiplication and s is given by

$$
s=\max (|g 1 * * \mathrm{es}|,|g 2 * * \mathrm{es}|)
$$

Where, $g 1=\left[\begin{array}{lll}-1 & 0 & 1\end{array}\right], g 2=$ transpose $\left[\begin{array}{lll}-1 & 0 & 1\end{array}\right]$, and “**" denotes two-dimensional linear convolution. The binary edge image (e) is then given by

$$
e= \begin{cases}0, & \text { otherwise } \\ 1, & \text { es }>\gamma\end{cases}
$$

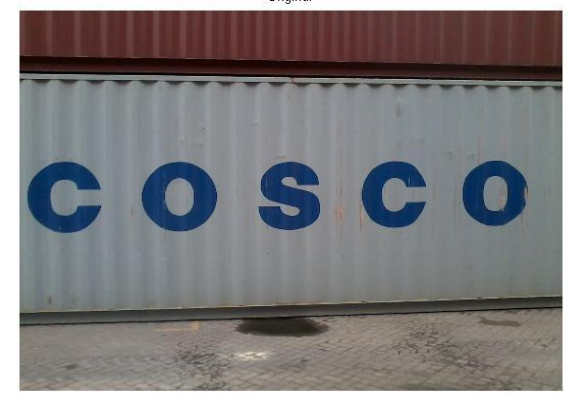

(a)

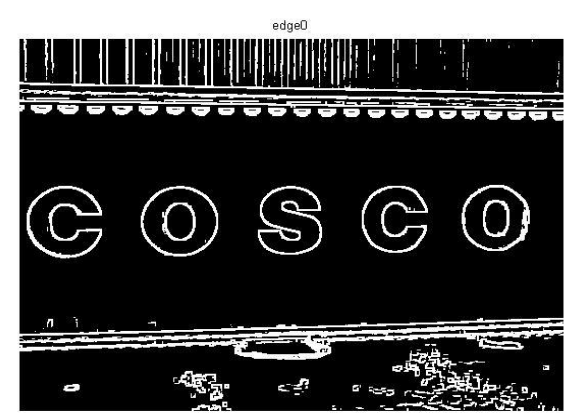

(b)

Fig 3: (a)Input Image,(b)Edge detection

\subsection{Close Operation}

Close edges in the binary edge image e are grouped by dilation using eight-connected structuring elements. Then, small connected components in the dilated image are filtered using erosion. The output is a binary image ec that contains text candidate regions.

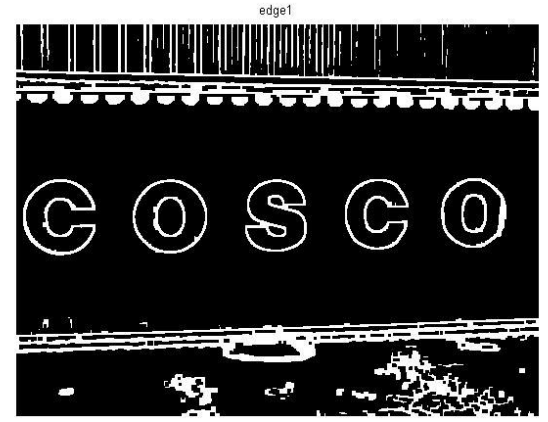

Fig 4:Close Operation

\subsection{Labelling of Text Regions}

All connected components of the edge image are screened with their position,size and area information.In our,algorithm we select connected components as a letter candidates if the following reqirements are met: 
1. $0.01<$ centre width of bounding box $<0.95$.

2. $0.2<$ centre height of bounding box $<0.8$.

3. Width vs.height ratio $<10$.

4. Width of the bounding box $<0.5$ image width and 10 pixels.

5. Height of the bounding box $>0.3$ image height and 30 pixels.

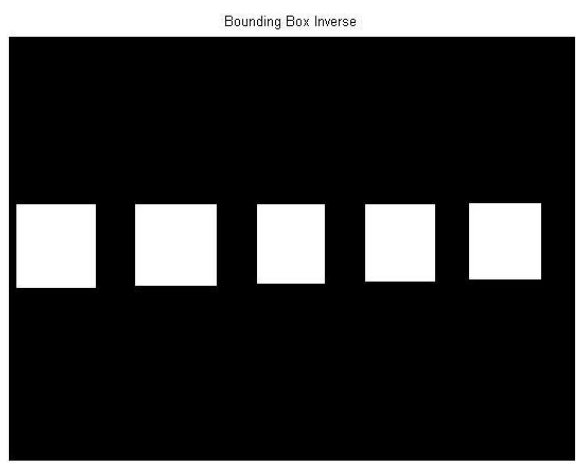

Fig 5:Text region filtering

\subsection{Text Region Binarization}

Obtain the threshold for the masked gray scale image for binarization.Theoritically after this step,only stroked letters are left as foreground 1 and rest of the image would go to background 0 .

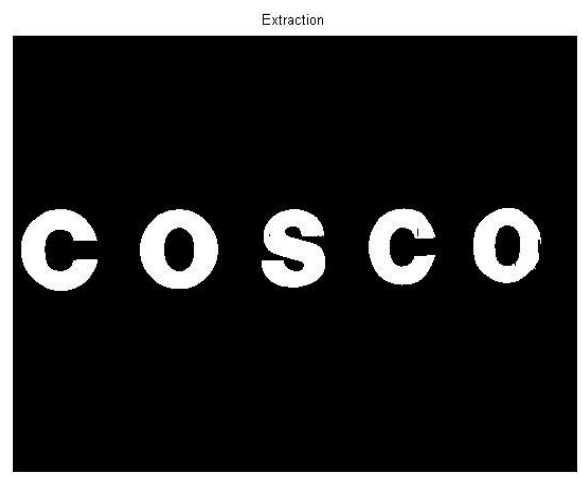

Fig 6:Text region binarization

\subsection{CHARACTER SEGMENTATION}

Scan the text region from top to bottom and left to right.If the full scanline is " 0 " then make it " 1 " otherwise do not make any change.For this method,there must be a space between two characters.

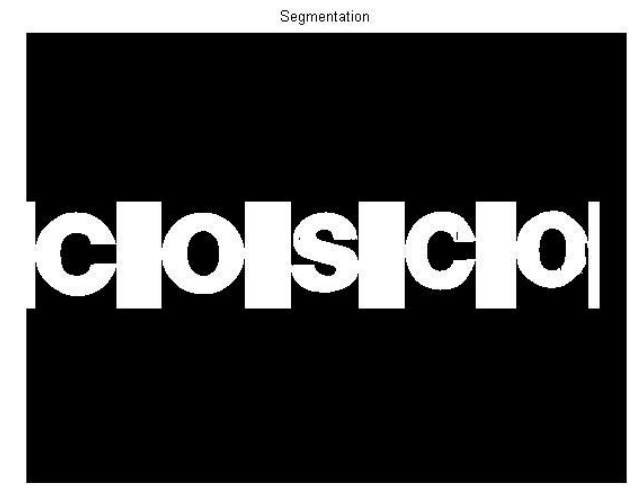

Fig 7:Character Segmentation

\section{EXPERIMENTAL RESULTS}

In order to evaluate our method,75 container images(1600x1200 pixels) obtained from real port environments were used in the experiment.The container names in the images are of varied colors and sizes and are aligned and located differently.

The extraction results are given in Table 1, which includes edge detection,labeling of text regions,character extraction,character segmentation and overall accuracy rate The accuracy rate for each module is defined as:

$$
\begin{gathered}
\text { Accuracy rate }=\frac{\text { Number of correctly processed samples }}{\text { Number of all test samples }} \\
\times 100 \%
\end{gathered}
$$

Table 1.Performance evaluation of proposed technique

\begin{tabular}{|l|l|}
\hline Module & Accuracy (\%) \\
\hline Edge Detection & 96 \\
\hline Labelling of text regions & 98.2 \\
\hline Character Extraction & 97.5 \\
\hline Character segmentation & 98 \\
\hline Overall performance & 97.4 \\
\hline
\end{tabular}

The overall accuracy rate is the product of accuracy rates of the four modules.

The proposed algorithm can accurately extract the text for varied positions,colors,illumination conditions,alignment modes and character sizes. 

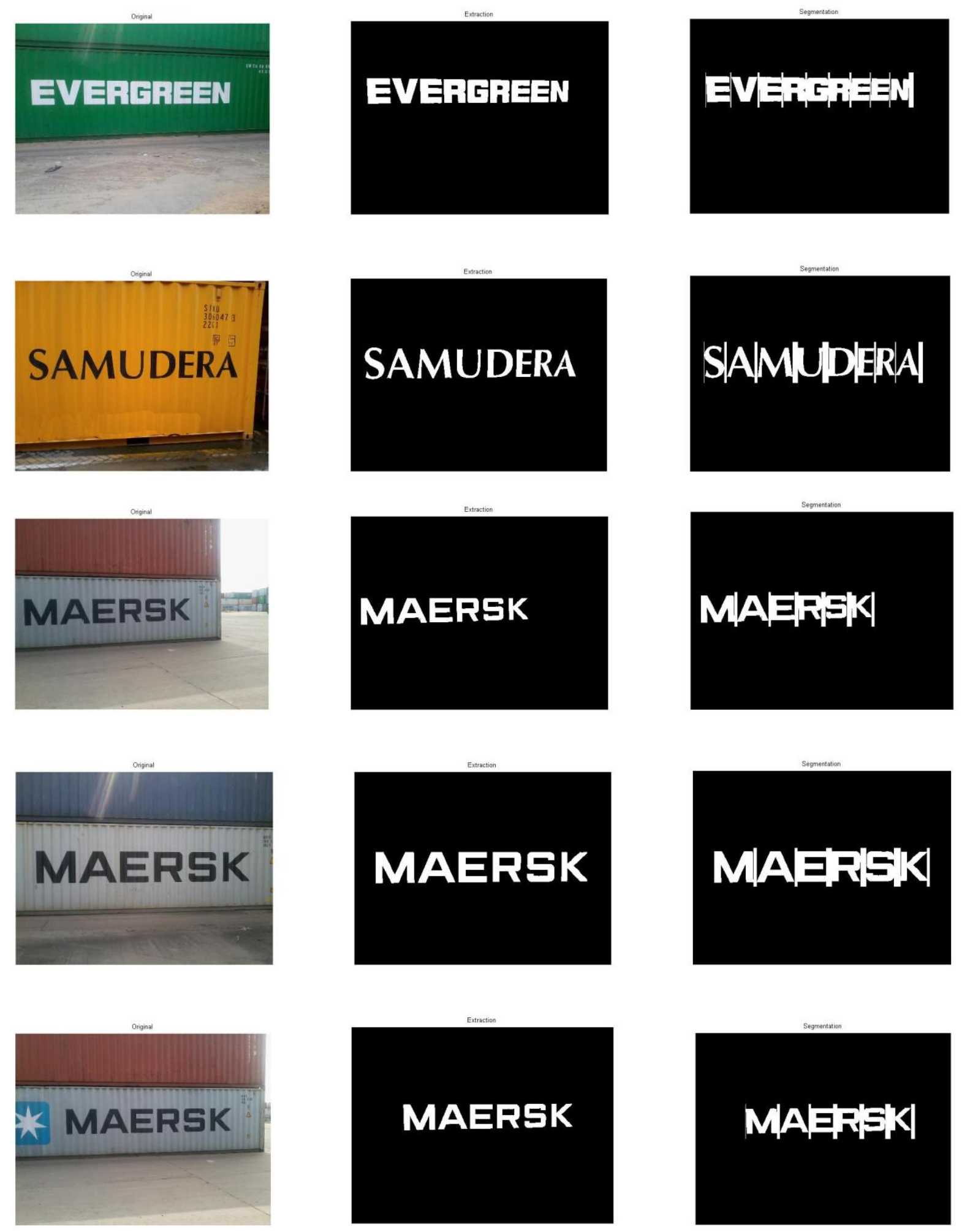

Fig 8:Some example of container name extraction and segmentation 


\section{COMPARISON WITH OTHER TEXT EXTRACTION TECHNIQUES}

To evaluate the performance there is a comparison with two existing algorithms [9] and [10].The first method has used a complicated method for finding inner,outer and inner-outer corners.

The second procedure has extracted text by identify edge at different orientation i.e 0,45,90,135 degrees and grouping these strokes at different heights.So it is difficult to identify the edges at different orientation.To solve the problems Conneted Component Variance(CCV) has used.

For finding a meaningfull word the isolated characters are grouped by dilation operation.The algorithm identifies each connected component and measures there properties.If the properties are not match with the properties in section 2.4 then it is symbol not character.

\section{CONCLUSION}

In this paper,we present a algorithm which is suitable to extract the container name of different colors,sizes and alignment modes.The experiments with container images captured from the real port environment demonstrate the effectiveness of the proposed technique.

Future work will focus on extracting container name from multiple images captured from the same container with one or multiple cameras during its moving. The container images are captured at the different positions or different time.

\section{REFERENCES}

[1] K. Jain and B. Yu, "Document representation and its application to page decomposition," IEEE Trans. Pattern Anal. Machine Intell., vol. 20, pp. 294-308, Mar. 1998.
[2] J. Ohya, A. Shio, and S. Akamatsu, "Recognizing characters in scene images," IEEE Trans. Pattern Anal. Machine Intell., vol. 16, pp. 215-220, Feb. 1994.

[3] H. M. Suen and J. F.Wang, "Text string extraction from images of colorpriented documents," Proc. Inst. Elect. Eng. Vis.,Image, Signal Process., vol. 143, no. 4, pp.210-216,1996.

[4] Nobuyuki Otsu, A threshold selection method from graylevel histograms. IEEE Trans.Sys.,Man., Cyber 9(1):62 66.

[5] Y. K. Ham, M. S. Kang, H. K. Chung, and R. H. Park, "Recognition of raised characters for automatic classification of rubber tires," Opt. Eng.,vol. 34, pp. 102108, Jan. 1995

[6] R. Lienhart, "Indexing and retrieval of digital video sequences based on automatic text recognition," in Proc. ACM Int. Conf., Boston, MA, Nov.1996.

[7] L. Wang and T. Pavlidis, "Direct gray-scale extraction of features for character recognition," IEEE Trans. Pattern Anal. Machine Intell., vol.15, pp. 1053-1067, Oct. 1993.

[8] Y. Zhong, K. Karu, and A. K. Jain, "Locating text in complex color images," Pattern Recognit., vol. 28, no. 10, pp. $1523-1535,1995$

[9] Jagath Samarabandu, Member, IEEE, and Xiaoqing Liu 2007 "An Edge-based Text Region Extraction Algorithm for Indoor Mobile Robot Navigation" ,International Journal of Signal Processing 3(4 )2007.

[10] Yassin M. Y. Hasan and Lina J. Karam 2000 "Morphological Text Extraction from Images" IEEE Transaction on Image Processing vol. 9,no.11,Nov.2000 